\title{
cDNA Microarray Analysis of Adaptive Changes after Renal Ablation in a Sclerosis-Resistant Mouse Strain
}

\author{
Brigitta Rumberger $^{a}$ Oliver Vonend ${ }^{d}$ Clemens Kreutz ${ }^{b}$ Jochen Wilpert ${ }^{a}$ \\ Johannes Donauer ${ }^{a}$ Kerstin Amann ${ }^{\mathrm{e}}$ Rolf Rohrbach ${ }^{\mathrm{c}}$ Jens Timmer ${ }^{\mathrm{b}}$ \\ Gerd Walz $^{\mathrm{a}}$ Peter Gerke ${ }^{\mathrm{a}}$ \\ ${ }^{a}$ Renal Division, Department of Medicine, University Hospital Freiburg, ${ }^{b}$ Freiburg Center for Data Analysis and \\ Modeling, Department of Physics, University of Freiburg, and ' Department of Pathology, University of Freiburg, \\ Freiburg, ${ }^{d}$ Department of Nephrology, Marienhospital Herne, University of Bochum, Bochum, and \\ eDepartment of Pathology, University of Erlangen-Nürnberg, Erlangen, Germany
}

\section{Key Words}

Remnant kidney $\cdot$ Renal fibrosis $\cdot$ Transforming growth factor- $\beta_{1} \cdot$ Oxidative stress $\cdot$ Matrix metalloproteinases . ADAMTS $\cdot$ Tissue inhibitors of metalloproteinases

\begin{abstract}
Background: $5 / 6$ nephrectomy (Nx) in susceptible animals causes glomerular sclerosis and interstitial fibrosis in the remnant kidney. Oxidative stress, transforming growth factor- $\beta$ (TGF- $\beta$ ), and the de novo synthesis of collagen seem to contribute to this process. However, these factors might also be required for tissue repair without fibrosis. Methods: We examined dynamic changes after nephron loss in a mouse strain capable of complete recovery. C57BL/6 mice underwent single-session $\mathrm{Nx}$ and were followed for 40 weeks. Gene expression was monitored over 20 days using 22,000 cDNA microarrays. Results: The mice developed transient hypertension and glomerular hypertrophy after $\mathrm{Nx}$ but failed to progress to glomerular sclerosis or renal failure. Gene expression profiles revealed three stages of recovery, an early phase of injury response, an intermediate phase of extracellular matrix (ECM) production and a later phase of reconstitution. Surprisingly, oxidative stress responses and
\end{abstract}

collagen production were strongly upregulated soon after Nx. Furthermore, TGF- $\beta_{1}$ and connective tissue growth factor were rapidly upregulated and remained elevated. Conclusion: We suggest that oxidative stress, collagen production, profibrotic growth factors and ECM turnover are part of the comprehensive adaptation to nephron loss and not necessarily associated with progressive loss of renal function.

Copyright $\odot 2007$ S. Karger AG, Basel

\section{Introduction}

Progression of chronic renal disease remains a significant clinical problem. A limited renal insult with nephron loss commonly leads to glomerular hyperfiltration, followed by proteinuria, renal fibrosis and end-stage renal disease. Profibrotic growth factors like transforming growth factor- $\beta_{1}\left(\right.$ TGF- $\left.\beta_{1}\right)$ and connective tissue growth factor (CTGF) play a central role in this process [1]. They stimulate the expression of extracellular matrix (ECM) components, inhibit matrix-degrading enzymes and induce epithelial-mesenchymal transition (EMT), thus promoting renal fibrosis [2]. Release of these growth factors and the subsequent production of collagen have be-

\section{KARGER}

Fax +4161306 1234 E-Mail karger@karger.ch www.karger.com
(C) 2007 S. Karger AG, Basel

1420-4096/07/0306-0377\$23.50/0

Accessible online at:

www.karger.com/kbr
Peter Gerke

Renal Division, University Hospital Freiburg

Hugstetterstrasse 55

DE-79106 Freiburg (Germany)

Tel. +49 761270 3401, Fax +49 761270 3232, E-Mail peter.gerke@uniklinik-freiburg.de 
come the central paradigm of progressive renal failure after renal ablation, diabetic nephropathy, and chronic transplant nephropathy.

The 5/6 nephrectomy ( $\mathrm{Nx}$ ) animal model is well established to study progressive renal disease. Most rodent strains are susceptible to renal ablation and undergo a typical course of events. An initial increase in singlenephron glomerular filtration rate leads to glomerular hypertrophy, followed by a decline in renal function and proteinuria within few months. Histology reveals glomerular sclerosis and progressive interstitial fibrosis. In contrast to most rats, several mouse strains recover from Nx without major sequelae [3]. Whereas 129/SV and FVB mice develop at least moderate proteinuria and renal fibrosis after $\mathrm{Nx}$, kidneys of C57BL/6 mice seem hardly affected.

We therefore used C57BL/6 mice to study the beneficial adaptive changes after nephron loss through partial nephrectomy. Besides functional and histological examination we used cDNA microarrays to determine a time course of gene transcription. Even in the absence of subsequent fibrosis or renal failure, we identified an increased transcription of profibrotic growth factors, oxidative stress response and ECM components as part of the healing process.

\section{Subjects and Methods}

\section{Animal Preparation}

Eight-week-old male C57BL/6 mice were obtained from Charles River (Kisslegg, Germany). The animals were housed at the Animal Research Facility of the University Hospital Freiburg, and had free access to food and water. Body weights were determined at the start of the protocol, the time of surgery, and the time of sacrifice. To synchronize adaptive responses, $\mathrm{Nx}$ was performed as a one-step procedure using 8 -week-old male mice. After removing the right kidney, the left kidney was decapsulated, and the upper and lower poles were resected under anesthesia with xylazine-ketamine intraperitoneally. Dissected tissues were weighted, and remnant kidney function and weight as well as mean arterial blood pressure were studied at various time points thereafter in 4 animals per time point. Proteinuria after 40 weeks was determined in 3 animals and compared with age-matched controls. Additionally, 3 mice per time point were sacrificed to collect remnant kidneys for RNA extraction as described below. The kidneys obtained from the heminephrectomies were used as the control samples at day 0 .

\section{Immunohistochemistry and Electron Microscopy}

Remnant kidneys were subject to retrograde perfusion fixation using 3\% glutaraldehyde and embedded in paraffin. Fourmicrometer sections were cut, deparaffinized and stained with hematoxylin/eosin or periodic acid-Schiff (PAS) to evaluate the degree of glomerular and tubulointerstitial changes. Immunohistochemistry was performed essentially as described [4]. The washing buffer was PBS containing $0.04 \%$ Triton X-100 and $0.35 \%$ DMSO. All incubations were performed in a moist chamber at $4^{\circ} \mathrm{C}$. Endogenous peroxidase activity was quenched with $0.3 \%$ $\mathrm{H}_{2} \mathrm{O}_{2}$ for $30 \mathrm{~min}$. Anti-MT-1/2 mAb was obtained from DAKO (Hamburg, Germany) and tissues were incubated at 1:100 overnight. M2 anti-FLAG antiserum was obtained from Sigma-Aldrich (Taufkirchen, Germany). For indirect immunoperoxidase staining we used the Vectastain ABC kit (Vector Laboratories, Burlingame, Calif., USA) following the manufacturer's protocol.

For electron microscopy, fixation was performed with $3 \%$ glutaraldehyde in $0.08 \mathrm{M}$ cacodylate buffer ( $\mathrm{pH} 7.4)$ for $2 \mathrm{~h}$. Slices were then rinsed in $0.1 \mathrm{M}$ cacodylate buffer ( $\mathrm{pH}$ 7.4). The tissue slices were postfixed in $2 \% \mathrm{OsO}_{4}$ in a veronal acetate buffer, and then dehydrated in a graded series of ethanols including en block staining $(1 \mathrm{~h})$ in $1 \%$ uranyl acetate at the $70 \%$ ethanol step. The specimens were then transferred to propylene oxide, and subsequently embedded in Epon-Araldite. Eighty-nanometer sections were cut with diamond knives, mounted on naked grids, and examined with a Philips CM 10 electron microscope.

\section{Glomerular Geometry}

Glomerular volumes were determined as described previously [5]. Briefly, kidneys were dissected into $1-\mu \mathrm{m}$ slices perpendicular to the longitudinal axis. By using area-weighted sampling, 10 small pieces were selected for embedding in Epon-Araldite. Remaining kidney slices were embedded in paraffin, yielding one representative section of each slice for morphometric and stereological investigations. By using systematic subsampling, a Zeiss eyepiece with a 100 -point grid (magnification, $\times 100$ and $\times 400$; Integrationsplatte II; Zeiss, Oberkochen, Germany), and the point-counting method $\left(P_{\mathrm{P}}=A_{\mathrm{A}}=V_{\mathrm{V}}\right)$, the volume densities of cortex, medulla, and glomeruli were determined. The glomerular number per area $\left(N_{\mathrm{A}}\right)$ was counted, and the total glomerular volume $\left(V_{\text {glom }}\right)$ was calculated by multiplying the volume density of glomeruli by the cortex volume $\left(V_{\text {Vglom }} \times V_{\mathrm{C}}\right)$. The glomerular number per volume $\left(N_{\mathrm{V}}\right)$ was determined using the following equation: $N_{\mathrm{V}}=k / \beta \times N_{\mathrm{A}}{ }^{1.5} / V_{\mathrm{V}}{ }^{0.5}$, with $k=1.03$ and $\beta=1.382$. The total glomerular number $\left(N_{\text {glom }}\right)$ was calculated by multiplying the glomerular number per volume $\left(N_{\mathrm{V}}\right)$ by the cortex volume $\left(V_{\mathrm{C}}\right)$. The mean glomerular volume $(v)$ was then derived from the total glomerular volume and the total number of glomeruli per kidney $\left(V_{\text {glom }} / N_{\text {glom }}\right)$.

Direct Systolic Blood Pressure Measurements and Urinalysis

Mice were anesthetized with pentobarbital (50 mg/kg, i.p.) and placed on a heated board to maintain body temperature at $37^{\circ} \mathrm{C}$. A polyethylene catheter was inserted into the bladder for urine collection. Systolic and diastolic blood pressures were obtained via the left femoral artery by using a strain-gauge blood pressure transducer. Mice were euthanatized after the experiment. Urinalysis was performed colorimetrically using the BioRad Protein Assay (Life Science, Munich, Germany) and the Sigma creatinine kit (Sigma-Aldrich, Munich, Germany).

Microarrays

Remnant kidneys were harvested from 3 animals at days 1, 2, $5,10,14$ and 20 after Nx, respectively. Gene expression was determined for each remnant kidney in comparison with reference 
mRNA, and the logarithmic level of mRNA expression of normal kidneys (day 0) was subtracted for each time point. cDNA microarrays were produced and processed essentially according to the Stanford protocol (http://cmgm.stanford.edu/pbrown). All genes were obtained as bacterial stocks from the National Institute of Aging (http://lgsun.grc.nia.nih.gov/cDNA/15k.html, http:// lgsun.grc.nia.nih.gov/cDNA/NIA_7_4k.html), kindly distributed by the MRC, Cambridge, UK [6, 7]. Plasmids were purified using the Qiagen 96-well Turbo Kit (Qiagen, Hilden, Germany), and inserts were purified by PCR using vector primers flanking the individual inserts (5'-CTG CAA GGC GAT TAA GTT GGG TAA C-3' and 5'-GTG AGC GGA TAA CAA TTT CAC ACA GGA AAC AGC-3'). PCR products were purified by ethanol precipitation, and resuspended in $\mathrm{H}_{2} \mathrm{O}$. Aliquots were dried and resuspended in $3 \times$ SSC to a final concentration of approximately 40 $\mathrm{ng} / \mu \mathrm{l}$. Printing was performed on aminosilane-coated slides (CMT-GAP II Slides, Corning, N.Y., USA), using an arrayer that was assembled according to specifications by the Stanford group using software kindly provided by Joe de Risi (http://cmgm.stanford.edu/pbrown).

\section{RNA Preparation}

Frozen tissues were homogenized in $4 \mathrm{M}$ guanidinium isothiocyanate with $0.72 \% \quad \beta$-mercaptoethanol using a polytron (PT-MR2100, Kinematica, Lucerne, Switzerland). Total RNA was subsequently purified on a cesium chloride gradient. After ethanol precipitation RNA was resuspended at a concentration of $0.4-4 \mu \mathrm{g} / \mu \mathrm{l}$, and stored at $-80^{\circ} \mathrm{C}$.

\section{Hybridization}

All hybridizations were performed in the presence of an equal amount of reference RNA (Stratagene, La Jolla, Calif., USA) as published [8]. Twenty-four micrograms of either tissue or reference RNA were transcribed into cDNA in the presence of Cy3- or Cy5-labeled dUTP, using Superscript II reverse transcriptase (Invitrogen, Carlsbad, Calif., USA). All other steps, including hybridization, were performed following the protocol published by P. Brown et al. (http://cmgm.stanford.edu/pbrown). A PCR purification kit (Qiagen) was used for cDNA purification.

\section{Semiquantitative RT-PCR}

Total RNA $(1 \mu \mathrm{g})$ from two remnant kidneys was purified for each time point with the RNase-free DNase set (Qiagen) and reversely transcribed into cDNAs using oligo (dT) 12-18 primers and Superscript II reverse transcriptase (Invitrogen). Each PCR was carried out with 1,4- $\beta$-galactosidase as internal control in each reaction vial. The following primers were used $\left(5^{\prime}-3^{\prime}\right)$ : $\beta$-galactosidase (BG071892): caggcacgtcgtattgaaaa, caaggctgcacactcctgta; TGF- $\beta_{1}$ : gtcagacattcgggaagcag, ctgccgtacaactccagtga; CTGF: caaagcagctgcaaatacca, ggccaaatgtgtcttccagt. The PCR was initiated by denaturation $\left(94^{\circ} \mathrm{C}, 5 \mathrm{~min}\right)$, followed by 28 cycles of denaturation $(30 \mathrm{~s})$, annealing $\left(1 \mathrm{~min}, 55^{\circ} \mathrm{C}\right)$, and elongation $(2 \mathrm{~min}$, $72^{\circ} \mathrm{C}$ ). PCR products were run on $3 \%$ agarose gels, and evaluated in relation to the corresponding $\beta$-galactosidase band using Scion Image software (Scion, Frederick, Md., USA). Array results were confirmed by RT-PCR for the following genes (data not shown): collagen 3A1 (BG086357), SPARC (BG064802), heme oxygenase1 (HO-1; BG077732), FN14 (BG080700), and isopentyl-diphosphate delta-isomerase (BG079889).

\section{Data Analysis}

Signal intensities were measured by an Axon 4000A scanner using GenePix 3.0 software (Axon Instruments, Union City, Calif., USA). Image and data files, array layout, and all relevant information according to the MIAME guidelines (Minimum Information about a Microarray Experiment) were transferred to the GeneTrafficDuo database (Microarray Data Management and Analysis Software, Iobion Informatics, La Jolla, Calif., USA). To exclude artifacts near background range, all spots were eliminated when sample intensity or reference intensity was less than 50 or less than the local background. Local background was subtracted from spot intensities. Normalization was performed by the locally weighted scatter plot smoother subgrid normalization method. Log ratios of each gene are transformed to a mean of zero. An analysis of variances (ANOVA) was applied to detect differentially expressed genes. The p values obtained were controlled for multiple testing (FDR $=$ false discovery rate). An adjusted $\mathrm{p}$ value smaller than 0.05 was considered significant. Agglomerative hierarchical clustering was performed using the R statistical software package (www.r-project.org). Correlation was used to measure the distances of genes or arrays. Gene expression time courses are grouped by $\mathrm{k}$-means clustering to find main patterns of regulation. To test for enrichment of Gene Ontology (GO) categories [9], the number of appearances of all GO functions of a group of interesting genes is compared with the number of appearances in a reference group. Fisher's exact test was applied and calculated $\mathrm{p}$ values are corrected for multiple testing.

\section{Results}

\section{Nx in C57BL/6 Mice Is Not Associated with Fibrosis, Glomerular Sclerosis or Proteinuria}

Figure 1 demonstrates the functional changes after $\mathrm{Nx}$ in C57BL/6 mice. The remnant kidneys rapidly enlarge, reaching a maximum weight after 9 weeks (fig. 1a). An increase in both systolic and diastolic blood pressure occurred after 1 week and was followed by a decline to the initial range within 2-4 months (fig. 1b). Mean glomerular volumes increased significantly after 1 week with a decline to baseline levels 9 weeks after $\mathrm{Nx}$ (fig. 1c). Similarly, urine flow was almost doubled 1 week after renal ablation, but declined to control levels after 16 weeks (fig. 1d). The gain in body weight was not different between operated and control animals. None of the animals developed proteinuria or renal failure. As demonstrated in figure 1e, urinary protein excretion in 3 mice examined 40 weeks after $\mathrm{Nx}$ was not different from agematched controls. Light and electron microscopy performed at various time points up to 40 weeks after $\mathrm{Nx}$ revealed occasional mild mesangial hypercellularity but no interstitial renal fibrosis or glomerular sclerosis (fig. 2a-c). 


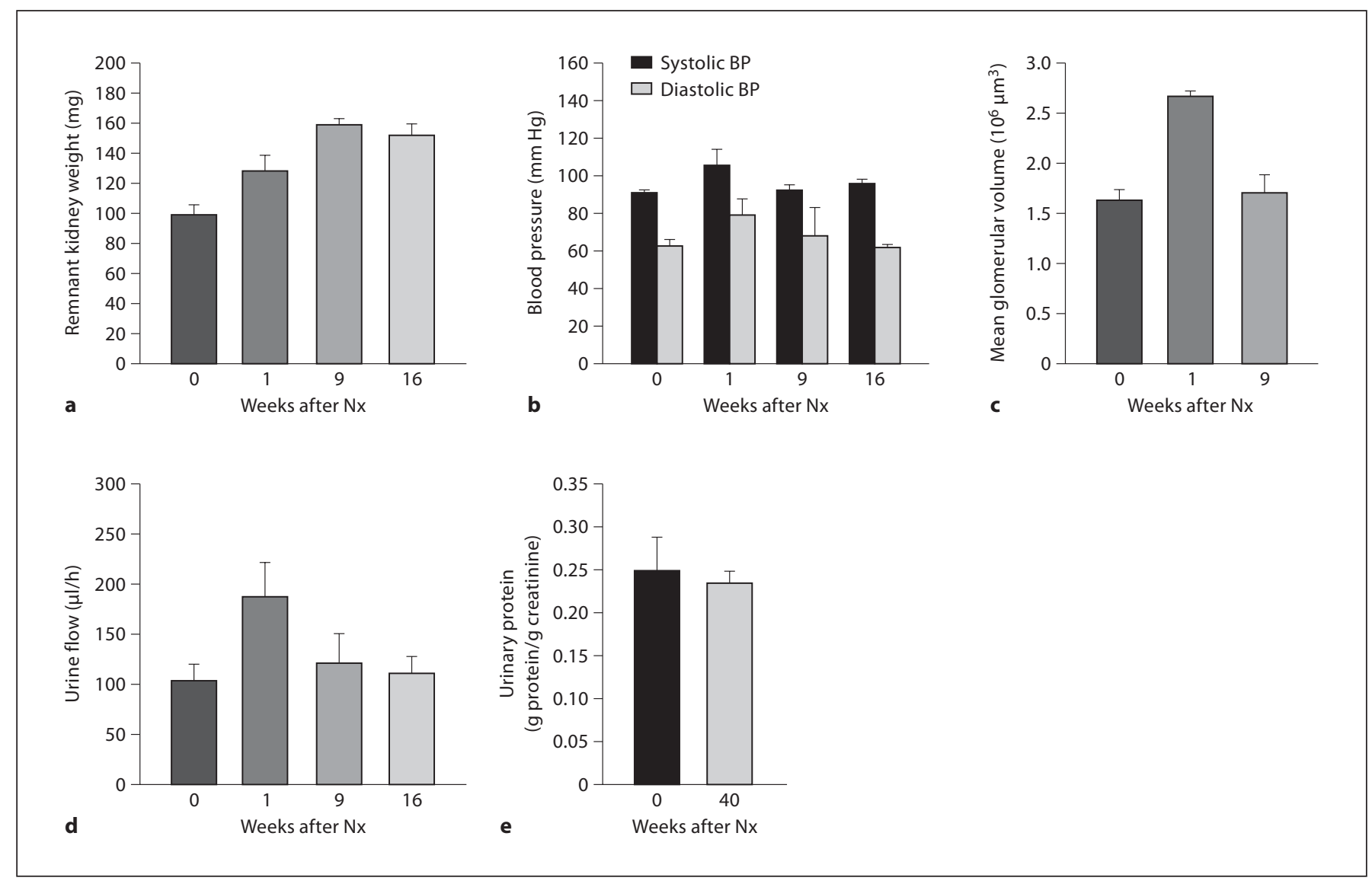

Fig. 1. Functional changes in C57BL/6 mice after Nx. a Remnant kidney weight increased rapidly after $\mathrm{Nx}$, peaking after 9 weeks. b There was a transient rise in blood pressure. However, both systolic and diastolic blood pressures (BP) approached baseline levels few weeks after Nx. c, d Mean glomerular volume and urine flow increased significantly after 1 week, and normalized within 9-16 weeks. e There was no difference in urinary protein excretion between mice 40 weeks after $\mathrm{Nx}$ and age-matched controls. Data are presented as mean \pm standard deviation.

\section{Transcriptional Analysis Defines Three Phases of}

Tissue Response

After selecting 176 significant genes by ANOVA all samples cluster according to their day of retrieval (fig. 3a). There are two major branches, one containing the samples from day 1 and day 2 and the second containing all the others. The latter has three branches. One is made up of controls (day 0 ) only, one exclusively contains day 5 samples and the third comprises days 10, 14 and 20.

\section{A Cluster of ECM Encoding Genes Shows Peak \\ Expression on Day 5}

Of the 176 genes identified by ANOVA to show a significant regulation over time after $\mathrm{Nx}, 52$ had peak expression levels on day 5 (fig. 3b). We suspected day 5 to be a turning point between early acute-phase response and late successful reconstitution. Therefore, gene ontology analysis was performed comparing these 52 genes with the entire array. Among functional groups significantly overrepresented were ECM constituents, collagens and cell adhesion molecules (table 1).

\section{Transcription of Metalloproteinases and Their Inhibitors}

Matrix accumulation is controlled by extracellular proteinases, in particular matrix metalloproteinases (MMP) and ADAMTS (a disintegrin and metalloproteinase with thrombospondin motifs), and by their inhibitors tissue inhibitors of metalloproteinases (TMP). As demonstrated in figure $4 \mathrm{a}$, collagen expression increased up to 28-fold after Nx. Maximal levels were reached on day 5. Figure $4 \mathrm{~b}$ demonstrates a significant upregulation of 


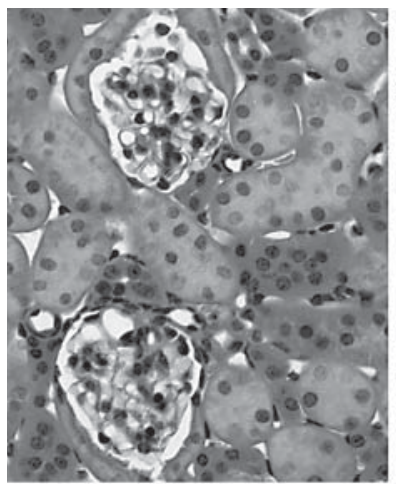

0

a

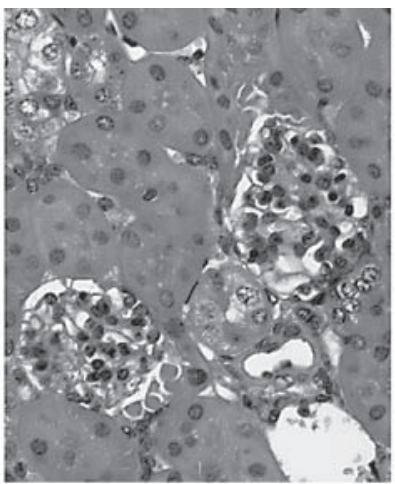

1

Weeks after $\mathrm{Nx}$

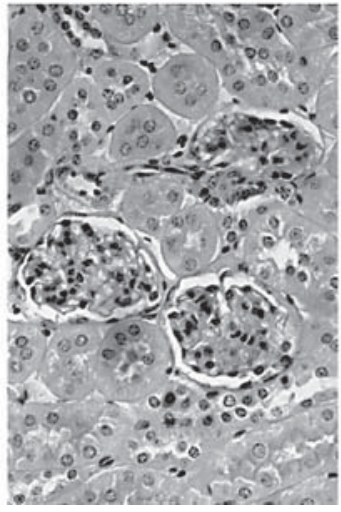

40 weeks after $\mathrm{Nx}$

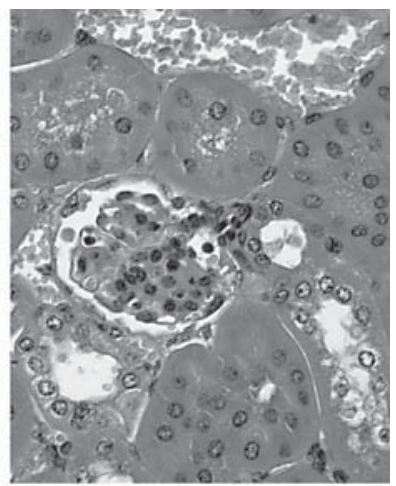

9

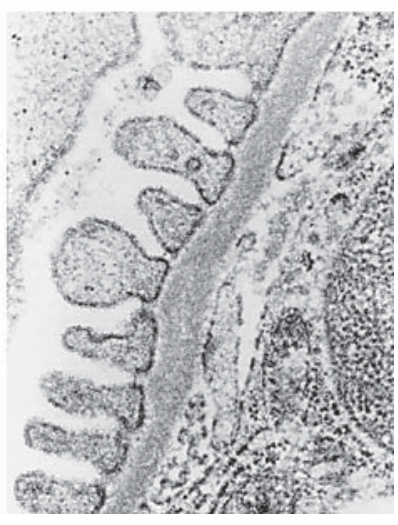

40 weeks after $\mathrm{Nx}$ c in C57BL/6. a Glutaraldehyde-fixed paraffin sections from remnant kidneys. The glomeruli showed a mild degree of mesangial hypercellularity after 9 weeks; the tubulointerstitial compartment appears unchanged. HE. $\times 120$. b Slides of remnant months after $\mathrm{Nx}$ and agematched controls. There was no obvious increase in ECM deposition in the remdney. PAS stain. Original magnifi cation $\times 120$. c Electron micrograph of a remnant kidney 9 months after Nx. Both membrane and podocyte foot any changes suggestive of glomerulosclerosis. Original magnification $\times 27,500$.

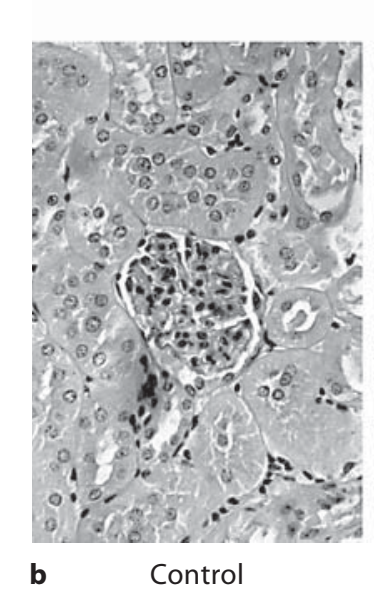

b Control
MMP-2, MMP-7 and MMP-23, whereas MMP-11 and MMP-9 transcription was not significantly altered after Nx. The MMP inhibitors TIMP-2 and TIMP-4 are also upregulated after Nx peaking on days 5 and 2, respectively (fig. 4d).

ADAMTS- 1 and ADAMTS-4 were strongly upregulated (fig. 4c), accompanied by a profound decrease in the transcription of their main inhibitor TIMP-3 (fig. 4d). A mild increase was evident for ADAMTS-2, ADAMTS-10 and ADAMTS-12. ADAMTS-19 transcription, on the other hand, was downregulated after Nx (fig. 4c).

\section{Early and Sustained Upregulation of TGF- $\beta_{1}$ and CTGF}

TGF- $\beta_{1}$ has been regarded as the key mediator of fibrogenesis with pleiotropic effects leading to ECM accumulation. Semiquantitative RT-PCR revealed that TGF$\beta_{1}$ is rapidly upregulated after Nx. TGF- $\beta_{1}$ expression reached a maximum on day 5 and remained elevated (fig. 5a, b). The expression of CTGF, another potentially profibrotic factor, followed a similar time course. In addition, we noted a prolonged upregulation of proteins that regulate TGF- $\beta_{1}$ action, including the cell surface TGF- $\beta$-binding protein betaglycan, decorin and biglycan (fig. 5c).

\section{Oxidative Stress Responses}

Among the phase 2 enzymes, the expression of microsomal glutathione S-transferase 1 (mGST1) and glutathione S-transferase omega 1 (GSTo1) were significantly upregulated reaching a maximum at day 1 (fig. 6a). Glutathione synthetase (GSS), on the other hand, was downregulated. Transcription of the other glutathione transferases and synthetases examined was not significantly altered after Nx. HO-1, the rate-limiting enzyme in heme catabolism, was dramatically upregulated, reaching an 18 -fold maximum $24 \mathrm{~h}$ after renal ablation (fig. 6b). As expected, the expression of the constitutively expressed heme oxygenase-2 (HO-2) remained unchanged. There was also an early upregulation of aldo-keto reductase 


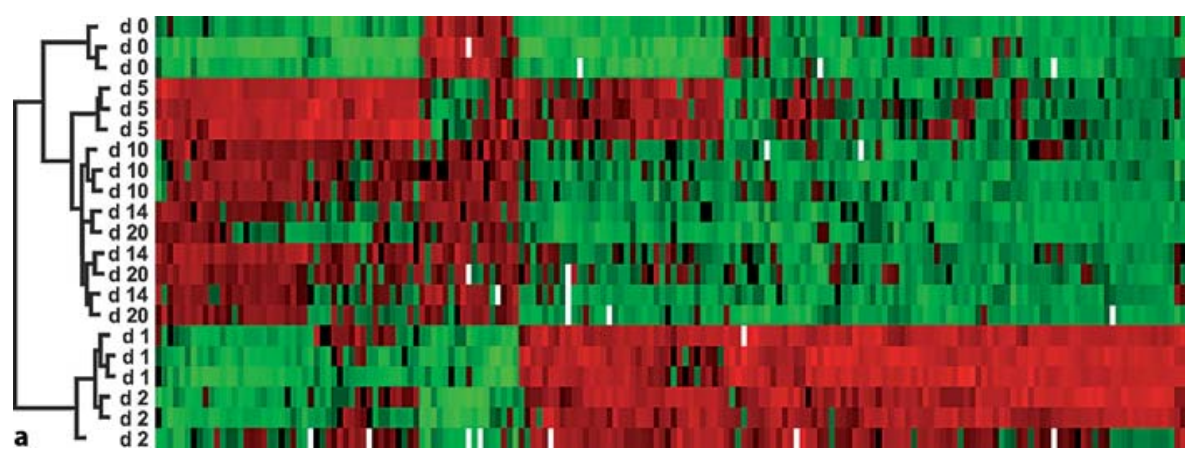

Fig. 3. Cluster analysis of transcriptional changes over 20 days after Nx. a Agglomerative hierarchical cluster analysis of individual arrays, based on an ANOVA on a significance level of $<0.05$. 176 genes fulfilled the criteria and are shown in columns, samples are sorted in rows. Light shades represent upregulation, shades of dark downregulation. Arrays cluster according to the day of retrieval (d). Four larger groups can be observed (day 0, days 1-2, day 5, and days 10-20). b Pattern of genes maximally regulated on day 5 after Nx. Time course of 52 significantly regulated genes with a maximum expression on day 5 after Nx. The black line indicates the average expression pattern of these genes, vertical bars indicate standard deviations.

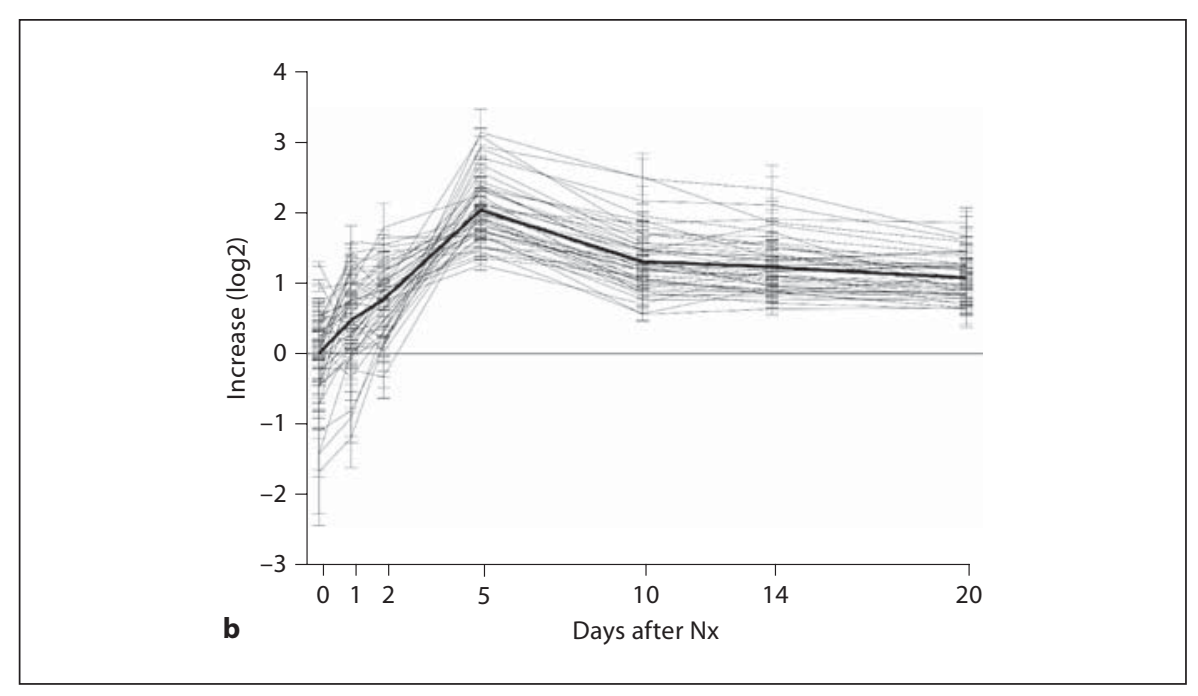

family 1, member B8 (Akrlb8), whereas other members of this family were downregulated.

$\mathrm{Cu}-\mathrm{Zn}$ superoxide dismutase (SOD) 1 and 2 were not significantly regulated after renal ablation (fig. 6c). In contrast, glutathione reductase (GSR) and thioredoxin reductase (Txnrd1) were increased. Glutathione peroxidase Gpx 1 decreased, reaching a minimum at day 2 after Nx. Metallothionein-2 (MT-2) was among the antioxidants regulated most significantly reaching a 12 -fold increase shortly after renal ablation (fig. 6d). Interestingly, leukemia inhibitory factor (LIF), a factor involved in mesenchymal-to-epithelial conversion of renal tubular cells and known to induce MT-2, was also upregulated after $\mathrm{Nx}$, closely following the time course of MT-2 (fig. 6d). The increase in MT-2 transcription was associated with increased translation as shown by immunohistochemistry (fig. 6e).

\section{Discussion}

Transcriptome analysis using microarray technology represents a powerful tool to study mechanisms of disease as well as physiologic adaptive changes. This technique has been used in numerous mouse models of progressive renal disease and helped to identify profibrotic factors and prognostic markers. However, some of these factors might also be involved in the healing process after kidney injury. To clarify this, we addressed the dynamic response in a mouse strain capable of recovering from $\mathrm{Nx}$ without developing renal fibrosis or proteinuria.

In our C57BL/6 mice, there was a gain in remnant kidney weight after $\mathrm{Nx}$ peaking around week 9 . Similar findings have been described in other mouse and rat models and are attributed to renal hyperplasia and hypertrophy of the remaining nephrons. Systemic blood pressure increased temporarily but reached baseline levels within 9 weeks. A rise in blood pressure after Nx is typical, and attributed to the activation of the renal renin-angiotensin-aldosterone system. However, the subsequent nor- 
Table 1. Gene ontology functional categories most significantly overrepresented on day 5 after $\mathrm{Nx}$

\begin{tabular}{ll}
\hline Gene ontology category & p FDR \\
\hline ECM (sensu Metazoa) & 0.000000 \\
ECM structural constituent (tensile strength) & 0.000000 \\
Collagen & 0.000000 \\
ECM structural constituent & 0.000000 \\
Extracellular space & 0.000000 \\
Phosphate transport & 0.000000 \\
Structural molecule activity & 0.000002 \\
Cell adhesion & 0.000028 \\
Calcium ion binding & 0.001000 \\
Dipeptidyl-peptidase I activity & 0.001173 \\
Positive regulation of type IIa hypersensitivity & 0.001596 \\
Lysosome & 0.001716 \\
Mitotic spindle checkpoint & 0.001887 \\
Positive regulation of phagocytosis & 0.002979 \\
Actin binding & 0.002979 \\
Basement membrane & 0.004667 \\
Complement activation & 0.007108 \\
Membrane & 0.009607 \\
Cytoplasm & 0.016338 \\
Calmodulin binding & 0.016410 \\
\hline
\end{tabular}

Of the 176 genes identified by ANOVA to show a significant regulation over time after $\mathrm{Nx}, 52$ had peak expression levels on day 5 . These 52 genes were tested for enrichment of GO categories in relation to the entire array. The 20 functional categories most significantly overrepresented in this group and their adjusted $\mathrm{p}$ values are listed.

$\mathrm{FDR}=$ False discovery rate

malization in this mouse strain is rather unusual. Furthermore, we did not observe signs of renal fibrosis or glomerulosclerosis by light microscopy or electron microscopy within 40 weeks after $\mathrm{Nx}$, and there was no development of proteinuria. The absence of fibrotic changes confirms other studies suggesting that C57BL/6 mice do not develop long-term sequelae after subtotal renal ablation [3].

Using ANOVA and cluster analysis of differentially expressed genes of remnant kidneys collected during 20 days after $\mathrm{Nx}$, we found that samples clustered according to their day of retrieval. This finding indicates a universal response without major interindividual differences. The dendrogram supports the existence of three distinct phases in the adaptive response to Nx. An early acutephase reaction (days 1 and 2) is followed by a distinct intermediate state (around day 5) and a later phase of reconstitution (days 10-20). Transcription peaking on day 5 included mainly genes encoding ECM components such as collagens or fibronectin. Interestingly, they are downregulated again towards day 20 . This may be indicative of transient matrix production as required for tissue repair without excessive scarring or progressive fibrosis.

Abnormal ECM accumulation is a common finding in renal fibrosis, and altered matrix degradation is regarded as a key factor in this process. However, ECM deposition and turnover may also be beneficial and essential after tissue injury. We observed a delayed upregulation of MMP-2 transcription, a secreted metalloproteinase with pleiotropic effects. MMP-2 is an antifibrotic enzyme facilitating matrix degradation but has also been associated with renal scarring. It can degrade the tubular basement membrane thereby inducing EMT with resultant tubular atrophy and fibrosis [10]. Interestingly, we observed an upregulation of TIMP-2 transcription with an almost identical time course, suggesting that some of the functions of MMP-2 might be blocked locally on a posttranslational level. MMP-7 was also significantly upregulated during the entire study period of 20 days after Nx. While the gene is hardly expressed in normal kidneys, an upregulation has been observed in various models of irreversible renal fibrosis [11]. MMP-7 can cleave E-cadherin thereby facilitating epithelial reorganization and possibly EMT. In fact, increased levels of MMP-7 mRNA have been identified as a marker of poor prognosis in human hydronephrotic kidneys [12]. Nevertheless, our data suggest that this MMP might also have beneficial effects in tissue repair.

Transcription of the metalloproteinases ADAMTS-1 and ADAMTS- 4 was strongly upregulated peaking on day 1 after Nx. These enzymes are mainly responsible for degrading aggrecan, a process involved in cartilage destruction as seen in arthritides. However, aggrecan can also protect collagen fibrils from degradation by collagenases [13], so the function of these ADAMTS in our setting might be to facilitate collagen turnover. Interestingly, the gene of their main inhibitor TIMP-3 was inversely regulated, possibly enhancing their effect on ECM degradation.

The growth factors TGF- $\beta_{1}$ and CTGF have been identified as key players in the development of renal fibrosis in various human diseases and animal models. Surprisingly, we also found a profound upregulation of their transcription in our model of successful recovery from nephron loss. Although ectopic expression of TGF- $\beta_{1}$ causes renal fibrosis [14], TGF- $\beta_{1}$ is a pleiotropic growth factor and the cellular effects may depend on its spatial and temporal activation. Interestingly, TGF- $\beta_{1}$ expression after $\mathrm{Nx}$ was accompanied by a prolonged upregula- 


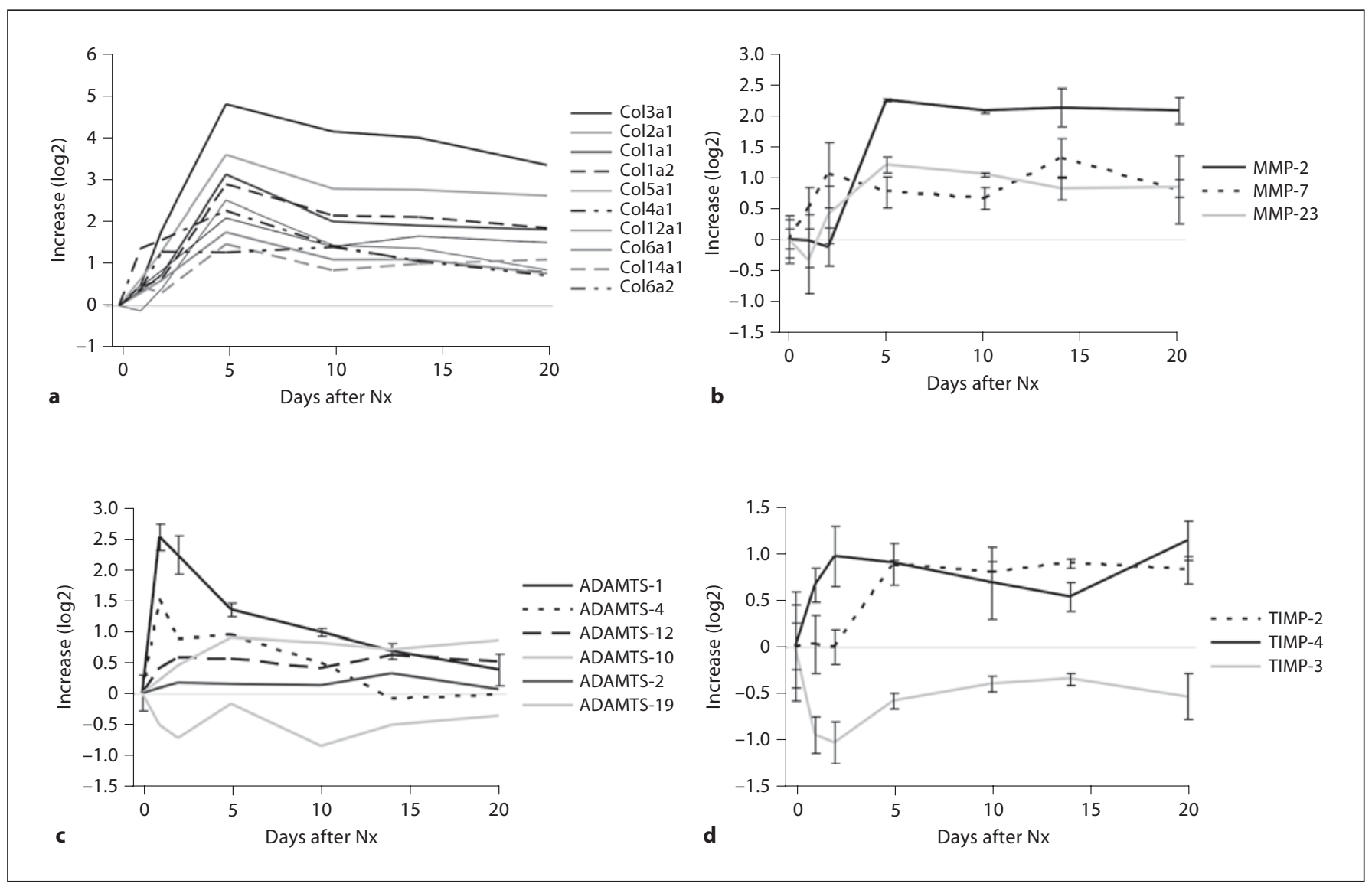

Fig. 4. Transcriptional changes in metalloproteinases and their inhibitors after Nx. a Collagen expression increased profoundly after Nx reaching a maximum on day 5 . Collagen type III alpha 1 (Col3a1) showed the highest expression level (30-fold). b Upregulation of MMP. Expression of MMP-2 and MMP-23 is upregulated after Nx peaking on day 5 and remains increased throughout the study period. MMP-7 is also increased peaking on days 2 and 14 after Nx. c Changes in ADAMTS. There is a significant

tion of proteins that regulate its action, such as betaglycan, decorin, and biglycan. Betaglycan has been reported to inhibit TGF- $\beta_{1}$ signaling in renal cells by preventing the dimerization of TGF- $\beta$ receptors, and both decorin and biglycan have been demonstrated to inactivate TGF$\beta_{1}$ [15]. In addition, decorin can lower glomerular levels of TGF- $\beta_{1}$, and ameliorate ECM accumulation [16]. Thus, expression of TGF- $\beta_{1}$ alone does not necessarily predict or cause renal fibrosis, but may require the permissive effects of regulatory partners to inflict renal damage.

CTGF mRNA expression is increased in tubular epithelial cells in a variety of chronic renal diseases and has been regarded a promising target of antifibrotic therapy [1]. In subtotally nephrectomized TGF- $\beta_{1}$ transgenic

early upregulation of ADAMTS-1 and ADAMTS-4 (day 1), followed by an increase in ADAMTS-10 (days 5-20). Expression of ADAMTS-19, on the other hand, is slightly downregulated. d Changes in TIMP. Whereas TIMP-2 and TIMP-4 were upregulated peaking on days 5 and 2, respectively, TIMP-3 was downregulated early after $\mathrm{Nx}$ and remained low throughout the study period.

mice the intravenous administration of CTGF antisense oligodeoxynucleotide from day 29 to 56 significantly reduced levels of CTGF mRNA in the tubular epithelium and limited renal interstitial fibrogenesis [2]. Our data, however, suggest that an early upregulation of this gene is not necessarily associated with renal fibrosis but might also be required for tissue repair.

Oxidative stress during the early adaptive phase after $\mathrm{Nx}$ has been implicated in the pathogenesis of long-term glomerular sclerosis, and therapy with antioxidants can ameliorate the course of renal failure after renal ablation [17]. Reactive oxygen species produced by an increased metabolism and oxidative phosphorylation after renal injury triggers TGF- $\beta$, thereby providing a direct link be- 


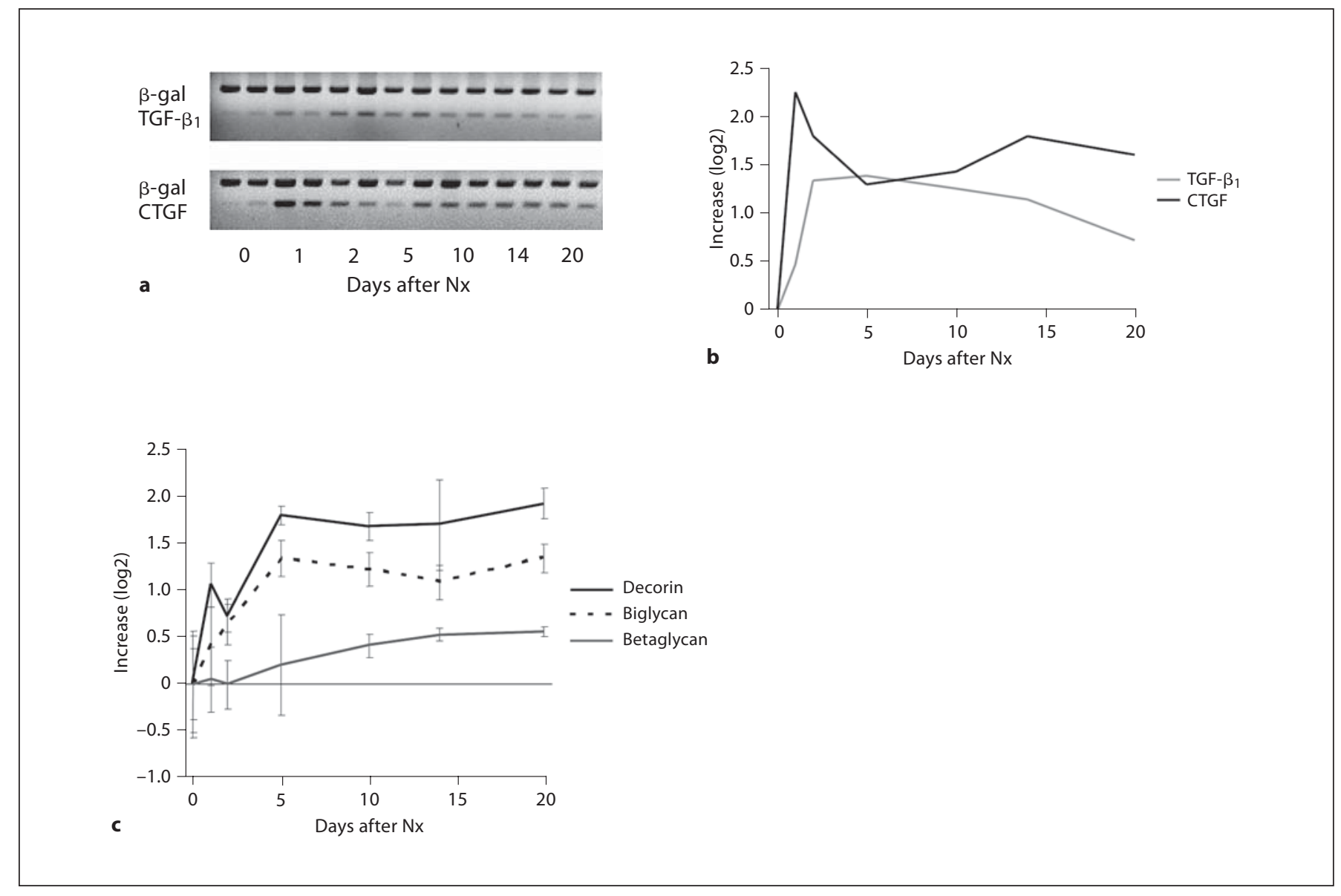

Fig. 5. Upregulation of profibrotic growth factors after Nx. a RTPCR products for TGF- $\beta_{1}$ and CTGF genes. PCR was performed on RNA from 2 animals for each gene and time point. $\beta$-Galactosidase ( $\beta$-gal), a gene not differentially regulated after $\mathrm{Nx}$, was used as an internal control. One example for each PCR reaction is shown; the lanes correspond to day 0, 1, 2, 5, 10, 14, 20 (from left to right in duplicate). $\mathbf{b}$ Normalization of the data shown in a demonstrates increased levels of both growth factors after Nx. Expression of both genes showed an early peak (CTGF on day 1 , TGF- $\beta_{1}$ on days $2-5$ ), and slightly decreased again towards day 20 . The graphic depicts the ratios of gene of interest and $\beta$-galactosidase over the time course (medians of the duplicates from one experiment are shown). c Upregulation of small leucine-rich proteoglycans and betaglycan after Nx. Relative changes of mRNA expression were determined by cDNA microarray. The TGF- $\beta_{1}$-binding proteins decorin, biglycan and betaglycan were strongly upregulated after renal ablation. Changes were most pronounced for decorin, revealing a 4 -fold increase. The levels of all three genes remain elevated throughout the observation period. tween reactive oxygen species generation and renal fibrosis. Our analysis revealed that $\mathrm{Nx}$ induces a complex expression pattern of antioxidants. HO-1, MT-2, mGST1, GSTo1 and Txnrd1 were rapidly upregulated (day 1), followed by Akrlb8, GSR (day 2) and Gpx3 (day 5). Expression of GSS, Akrla4 and Gpx1, however, decreased early after Nx. HO-1 is triggered by several stimuli, including heme, hydrogen, cytokines, and growth factors. However, the precise molecular mechanism of its induction after Nx remains to be elucidated. It is interesting to note that dopamine triggers HO-1 in vitro, and favorably affects the outcome of cadaveric kidney transplantation [18]. Thus, it is possible that the rapid increase of HO-1 after $\mathrm{Nx}$ is mediated by adrenergic stimuli and/or changes in hemodynamics. Glutathione peroxidases are seleniumcontaining enzymes that catalyze the reduction of various hydroperoxides. A Gpx-like protein has been shown to represent a $\mathrm{H}_{2} \mathrm{O}_{2}$ sensor in Saccharomyces cerevisiae necessary for hydrogen peroxide homeostasis [19]. Hence, the generation of reactive oxygen species may act as a second messenger that induces a comprehensive antioxidant program to prevent further damage. MT-2 belongs to a 


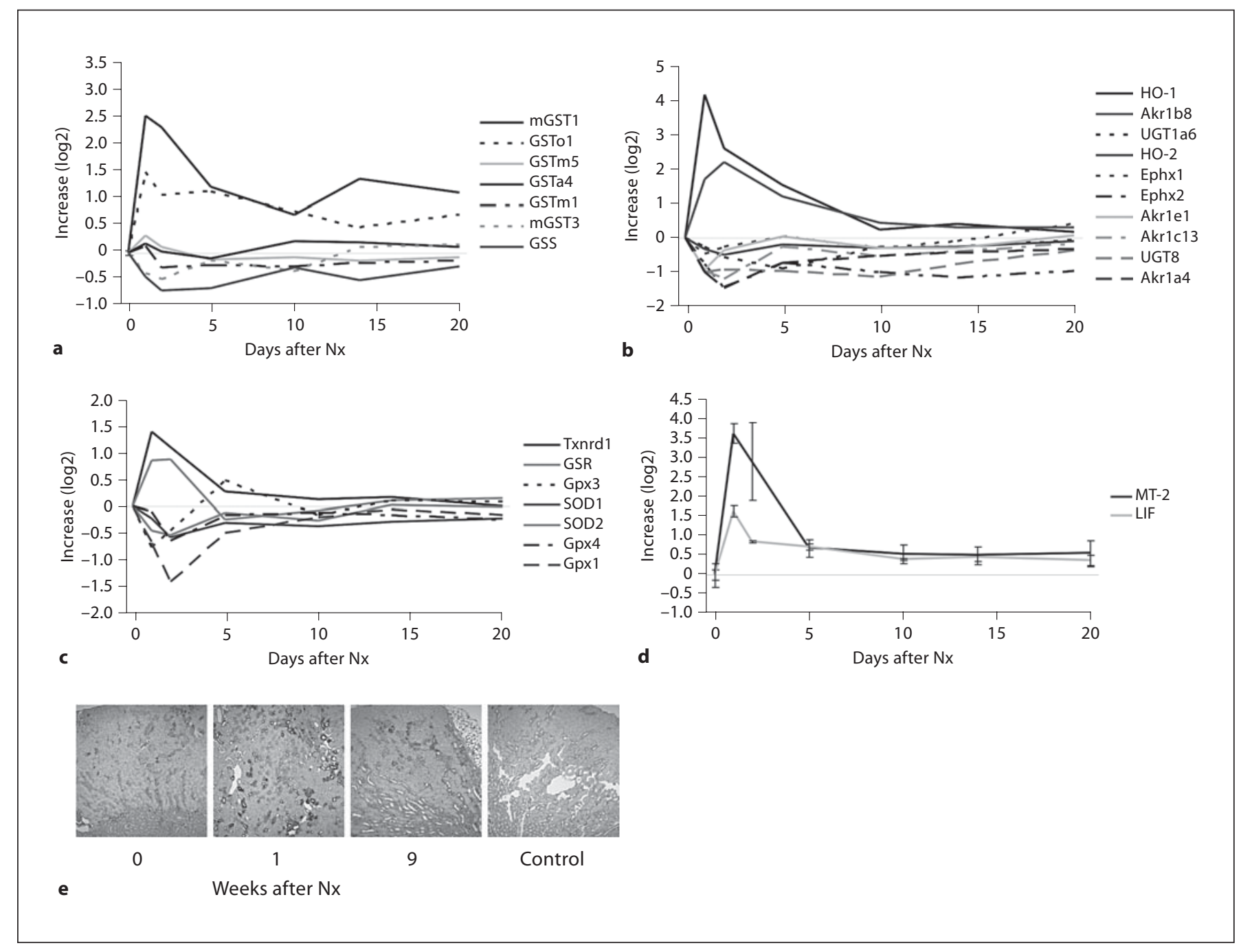

Fig. 6. Upregulation of antioxidant systems after Nx. a Upregulation of glutathione transferases. Expression of the mGST1 and the GSTol increased while other glutathione transferases and synthetases were basically unchanged or downregulated [GSS; GSTa4 (glutathione S-transferase alpha 4)]. b Upregulation of phase 2 enzymes after Nx. HO-1, but not HO-2, was rapidly upregulated after $\mathrm{Nx}$ and remained elevated until day 5. Akr1b8 followed a similar course, whereas Akrla4 was downregulated. The other phase 2 enzymes examined [UGT (UDP-glucuronosyltransferase); Ephx (epoxide hydrolase)] were unchanged or slightly downregulated after Nx. c Upregulation of other antioxidant systems

after Nx. SOD1 or SOD2 were not significantly regulated. In contrast, the GSR and Txnrd1 as well as the glutathione peroxidase Gpx3 were upregulated. It is interesting to note that Gpx3 expression peaks at day 5 after an early decrease. Gpx 1 decreased most significantly (day 2). d Upregulation of MT-2 and LIF after Nx. Expression of both MT-2 and LIF is rapidly upregulated after Nx and appears to follow a similar time course. e Anti-MT-2 staining in remnant kidneys on days 0, 7 and 63. MT-2 expression is seen as dark staining in the tubular epithelium. M2 anti-FLAG antiserum served as negative control (right, original magnification $\times 100)$.

superfamily of intracellular metal-binding proteins with unique redox capabilities. MT-2 is induced by zinc, hypoxia, and various cytokines, and accumulates in rapidly proliferating cells [20]. Interestingly, LIF, a factor involved in mesenchymal-to-epithelial conversion of tubular epithelial cells, closely follows the time course of MT-

2. Taken together, the sequential activation of different antioxidant systems suggests that Nx triggers a well-orchestrated cascade of signaling events that limit the damage caused by reactive oxygen species.

Our results show that oxidative stress responses, increased expression of TGF- $\beta_{1}$ and collagen production 
might be part of necessary adaptive mechanisms to subtotal renal ablation. Hence, even though the exact role of single genes and the influence of the genetic background and confounding factors such as hypertension remain elusive, they suggest that these factors are not unconditionally profibrotic but may be counteracted or might even be essential components of successful tissue repair.

These observations question several central paradigms of renal fibrosis: neither oxidative stress responses nor collagen production are necessarily associated with renal fibrosis. Most importantly, our findings suggest that an early increase in the expression of TGF- $\beta_{1}$, CTGF, MMPs or ECM components cannot be used to predict renal fibrosis or progressive renal failure in this mouse model.

\section{Acknowledgements}

We thank P. Stunz, T. Kilic and M. Klein for expert technical assistance and the members of the Walz laboratory for valuable discussion. This work was supported by DFG grant WA 597.

\section{References}

1 Zoja C, Abbate M, Remuzzi G: Progression of chronic kidney disease: insights from animal models. Curr Opin Nephrol Hypertens 2006;15:250-257.

2 Okada H, Kikuta T, Kobayashi T, Inoue T, Kanno Y, Takigawa M, Sugaya T, Kopp JB, Suzuki H: Connective tissue growth factor expressed in tubular epithelium plays a pivotal role in renal fibrogenesis. I Am Soc Nephrol 2005; 16:133-143

3 Ma LJ, Fogo AB: Model of robust induction of glomerulosclerosis in mice: Importance of genetic background. Kidney Int 2003;64: 350-355.

4 Gerke P, Huber TB, Sellin L, Benzing T, Walz G: Homodimerization and heterodimerization of the glomerular podocyte proteins nephrin and neph1. J Am Soc Nephrol 2003; 14:918-926.

5 Amann K, Irzyniec T, Mall G, Ritz E: The effect of enalapril on glomerular growth and glomerular lesions after subtotal nephrectomy in the rat: a stereological analysis. J Hypertens 1993;11:969-975.

6 Tanaka TS, Jaradat SA, Lim MK, Kargul GJ, Wang X, Grahovac MJ, Pantano S, Sano Y, Piao Y, Nagaraja R, Doi H, Wood WH 3rd, Becker KG, Ko MS: Genome-wide expression profiling of mid-gestation placenta and embryo using a 15,000 mouse developmental cDNA microarray. Proc Natl Acad Sci USA 2000;97:9127-9132.
7 VanBuren V, Piao Y, Dudekula DB, Qian Y, Carter MG, Martin PR, Stagg CA, Bassey UC, Aiba K, Hamatani T, Kargul GJ, Luo AG, Kelso J, Hide W, Ko MS: Assembly, verification, and initial annotation of the NIA mouse 7.4K cDNA clone set. Genome Res 2002;12: 1999-2003.

8 Boldrick JC, Alizadeh AA, Diehn M, Dudoit S, Liu CL, Belcher CE, Botstein D, Staudt LM, Brown PO, Relman DA: Stereotyped and specific gene expression programs in human innate immune responses to bacteria. Proc Natl Acad Sci USA 2002;99:972-977.

9 Ashburner M, Ball CA, Blake JA, Botstein D, Butler H, Cherry JM, Davis AP, Dolinski K, Dwight SS, Eppig JT, Harris MA, Hill DP, Issel-Tarver L, Kasarskis A, Lewis S, Matese JC, Richardson JE, Ringwald M, Rubin GM, Sherlock G: Gene ontology: tool for the unification of biology. The Gene Ontology Consortium. Nat Genet 2000;25:25-29.

10 Cheng S, Pollock AS, Mahimkar R, Olson JL, Lovett DH: Matrix metalloproteinase 2 and basement membrane integrity: a unifying mechanism for progressive renal injury. FASEB J 2006;20:1898-1900.

11 Nuttall RK, Sampieri CL, Pennington CJ, Gill SE, Schultz GA, Edwards DR: Expression analysis of the entire MMP and TIMP gene families during mouse tissue development. FEBS Lett 2004;563:129-134.

12 Henger A, Kretzler M, Doran P, Bonrouhi M, Schmid H, Kiss E, Cohen CD, Madden S, Porubsky S, Grone EF, Schlondorff D, Nelson PJ, Grone HJ: Gene expression fingerprints in human tubulointerstitial inflammation and fibrosis as prognostic markers of disease progression. Kidney Int 2004;65:904-917.
13 Pratta MA, Yao W, Decicco C, Tortorella MD, Liu RQ, Copeland RA, Magolda R, Newton RC, Trzaskos JM, Arner EC: Aggrecan protects cartilage collagen from proteolytic cleavage. J Biol Chem 2003;278:4553945545.

14 Piek E, Roberts AB: Suppressor and oncogenic roles of transforming growth factorbeta and its signaling pathways in tumorigenesis. Adv Cancer Res 2001;83:1-54.

15 Davies M, Kastner S, Thomas GJ: Proteoglycans: their possible role in renal fibrosis. Kidney Int 1996;54(suppl):S55-S60.

16 Isaka Y, Brees DK, Ikegaya K, Kaneda Y, Imai E, Noble NA, Border WA: Gene therapy by skeletal muscle expression of decorin prevents fibrotic disease in rat kidney. Nat Med 1996;2:418-423.

17 Amann K, Törnig J, Buzello M, Kuhlmann A, Gross ML, Adamczak M, Ritz E: Effect of antioxidant therapy with dl-alpha-tocopherol on cardiovascular structure in experimental renal failure. Kidney Int 2002;62: 877-884

18 Schnuelle P, Berger S, de Boer J, Persijn G, van der Woude FJ: Effects of catecholamine application to brain-dead donors on graft survival in solid organ transplantation. Transplantation 2001;72:455-463.

19 Inoue Y, Matsuda T, Sugiyama K, Izawa S, Kimura A: Genetic analysis of glutathione peroxidase in oxidative stress response of Saccharomyces cerevisiae. J Biol Chem 1999; 274:27002-27009.

20 Coyle P, Philcox JC, Carey LC, Rofe AM: Metallothionein: The multipurpose protein. Cell Mol Life Sci 2002;59:627-647. 